\title{
Correction to: A novel cost-effective sparsity-aware algorithm with Kalman-based gain for the identification of long acoustic impulse responses
}

\author{
Ayoub Tedjani $^{1}{ }^{1}$. Ahmed Benallal ${ }^{1}$
}

Published online: 18 August 2020

(c) Springer-Verlag London Ltd., part of Springer Nature 2020

\section{Correction to: Signal, Image and Video Processing https://doi.org/10.1007/s11760-020-01715-2}

Unfortunately, the above article has been published with some typos in it.

The corrected sentences are given below:

1. In $\mathrm{n}$ the subsection 4.1 Derivation of the proposed algorithm (IPNLMS), under part (b) Step 2: Simplifcation, the second line in fourth paragraph should read as:

This replacement is to set a fxed overall adaptation step-size independent on the infuence of the input signal power $\sigma_{x}^{2}$, and to have an update equation form comparable to IPNLMS

2. In equation (27), the zero symbol ' $\mathbf{0}$ ' in $\mathbf{0}_{N_{q} \times 1}$ should be written in bold because it refers to the vector of the first $N_{q}$ zero elements in $\mathbf{m}$. In addition, the elements of the vector should be spaced and separated, as shown below:

$$
\mathbf{m}_{N \times 1}=\left[\mathbf{0}_{N_{q} \times 1}, 1, e^{-1 / \phi}, e^{-2 / \phi}, \ldots, e^{-\left(N_{m}-1\right) / \phi}\right]^{T}
$$

3. In equation (28) the zero symbols ' 0 ' should be written in bold because they refer to the zero portions in the matrix $\mathbf{w}$. That is:

$$
\mathbf{w}=\left[\begin{array}{cc}
\mathbf{0}_{N_{q} \times N_{q}} & \mathbf{0}_{N_{q} \times N_{m}} \\
\mathbf{0}_{N_{m} \times N_{q}} & \mathbf{P}_{N_{m} \times N_{m}}
\end{array}\right] \mathbf{m}+\mathbf{q}
$$

Publisher's Note Springer Nature remains neutral with regard to jurisdictional claims in published maps and institutional affiliations.
The original article can be found online at https://doi.org/10.1007/ s11760-020-01715-2.

Ayoub Tedjani superayoub001@gmail.com

Ahmed Benallal

a_benallal@hotmail.com

1 Signal Processing and Image Laboratory (LATSI) Department of Electronics, Faculty of Technology, University of Blida 1, B.P. 270, Route de Soumâa, 09000 Blida, Algeria 